\title{
Cataract blindness and barriers to uptake of cataract surgery in a rural community of northern Nigeria
}

\author{
Mansur M Rabiu
}

\begin{abstract}
Aims-A population based cross sectional survey was conducted to determine the magnitude of cataract blindness and the barriers to uptake of cataract services in a rural community of northern Nigeria.

Methods-1461 people out of 1924 registered eligible people were examined. The study population was chosen by two stage cluster random sampling. In the first sampling stage 15 villages were randomly chosen while in the final stage 170 people who were 40 years and over were selected in each village. Each selected person had visual acuity recorded for both eyes. Those with vision of less than $3 / 60$ in the better eye were assessed for cataract. People with cataract were asked why they had not sought medical attention.

Results-A blindness prevalence of $8.2 \%$ (95\% CI $5.8 \%-10.5 \%)$ was found among the sampled population. Cataract was responsible for $44.2 \%$ of the blindness. Thus, a cataract blindness prevalence of $3.6 \%$ was found. The cataract surgical coverage (people) was $4.0 \%$ and the couching coverage (people) was $18 \%$. The main barrier to seeking cataract surgery was cost of the service $(61 \%)$.

Conclusion-Some regions of the world still have high burden of cataract blindness that needs attention. Such areas need an effective free cataract outreach programme.
\end{abstract}

(Br f Ophthalmol 2001;85:776-780)

Cataract is responsible for about 16 million blind people worldwide. The burden of blindness is more in remote rural communities of developing countries. ${ }^{1}$ These countries are characterised by high backlog of unoperated cataract and increasing incidence of cataract due to population ageing. However, these countries have inadequate and inefficient cataract surgical service. For these regions of the world, it becomes imperative to develop a cost effective, sustainable service delivery system for cataract surgery. However, this is only possible with relevant data on the magnitude of the cataract blind in the areas and also information on factors that hinder the people from accessing such sight restoring services in the areas. This is important because various studies $^{2}$ had revealed that availability of eye care services in such communities may not necessarily translate into appropriate utilisation.

There has evolved a rapid form of cataract blindness assessment in communities. ${ }^{3}$ This involves surveying the most vulnerable portion of the community - that is, the elderly, since cataract blindness (senile cataract) occurs mainly in old age.

A study was planned to determine the number of cataract blind and the barriers to cataract surgery uptake among people 40 years and above in a rural community of northern Nigeria. The study was also to determine the cataract surgical and couching coverage and to assess the visual outcome of cataract surgery and couching in the area.

\section{Materials and methods}

The study was a population based cross sectional survey of people 40 years and above in a rural community of Katsina State, northern Nigeria and was conducted in the months of April and May 1999.

\section{SAMPLE SIZE DETERMINATION}

The SAMPLE XS computer program using the following parameters and assumptions determined the minimum sample size.

- Target population of people 40 years and older $=23000$

This was based on estimates that $20 \%$ of the overall population $(112000)^{4}$ is 40 years of age or older. Census data by age group were not available.

- Prevalence of cataract blindness in people 40 years of age and older $=1.7 \%$

Previous blindness research in a nearby subdistrict indicated that the all age blindness prevalence rate was $1.14 \% .^{5}$ For the purposes of this study, it was assumed that the prevalence of blindness in people 40 years and older can be approximated by multiplying the overall population blindness prevalence by a factor of three. In addition, it was assumed that approximately one half of blindness in over 40 year olds would be attributed to cataract. As 
such, the estimated prevalence of cataract blindness in people above 40 years was assumed to be of the order of $1.7 \%$

- Design effect (due to cluster sampling strategy) $=1.5$

- Maximum error $=0.6 \%$

- Calculated minimum sample size $=2396$ subjects.

\section{STUDY DESIGN}

The study population was chosen by two stage cluster random sampling. In the first sampling stage 15 villages were randomly selected from the sampling frame of the 122 villages in the local government area (LGA). The selection was done using probability proportional to size (PPS). In the final sampling stage 170 people aged 40 years and older were chosen in each of the clusters (villages). They were chosen by randomly selecting a direction in the village and examining all eligible people in the households along that direction. This was continued until the required 170 people had been examined. All eligible people in the households either present or absent during the survey were registered.

\section{DATA COLLECTION PROCEDURES}

The data were collected in three stages. The first stage involved the registration of eligible people, the second stage was visual acuity testing, while the third stage involved eye examination. In the first stage for each selected person the personal data of name, age, sex, and occupation were recorded. Also each individual was asked if he/she ever had cataract surgery or couching procedures in the eye(s). This was confirmed by examining the eye for aphakia, using a pen torch. In the second stage, for each selected subject the presenting visual acuity (VA) of each eye was tested. The VA was measured with a modified Snellen's "E" tumbling chart. All aphakic people presenting with no aphakic glasses had their VA tested with +10 dioptre glasses.

All individuals with vision less than $3 / 60$ in one or both eyes proceeded to the third stage while those with vision of $3 / 60$ or better were not examined further. The first and second stages were conducted by ophthalmic nurses.

The third stage of data collection was conducted by the ophthalmologist. Using a pen torch and direct ophthalmoscope, the eyes were examined for the likely principal cause of blindness in the eyes. They were specifically examined for cataract, trachoma, glaucoma, post-intervention complication, or uncorrected aphakia (see operational definitions below) as a principal cause of the blindness. Individuals

Table 1 Age-sex distribution of sampled population (by 10 years age groups)

\begin{tabular}{lccc}
\hline Age groups (years) & Males (\%) & Females (\%) & Total (\%) \\
\hline $40-49$ & $228(36.9)$ & $389(63.1)$ & $617(42.2)$ \\
$50-59$ & $164(41.9)$ & $227(58.1)$ & $391(26.7)$ \\
$60-69$ & $155(52.0)$ & $143(48)$ & $298(20.4)$ \\
$70-69$ & $88(61.9)$ & $54(38.1)$ & $142(9.7)$ \\
$80+$ & $8(61.5)$ & $5(38.5)$ & $13(0.9)$ \\
Total & $643(44)$ & $818(56)$ & $1461(100)$ \\
\hline
\end{tabular}

with post-intervention complications were further examined to detect the main form of complication in the eye likely to explain the poor vision after the intervention (couching or cataract surgery). The people with cataract or couching in one or both eyes were asked why they had not had cataract surgery. The people with cataract and other treatable blindness were referred for treatment. All information was recorded in a specially designed questionnaire developed for the purposes of this survey.

\section{OPERATIONAL DEFINITIONS}

For the purpose of this survey the following definitions were applied.

- Blindness: presenting visual acuity of less than $3 / 60$ in the better eye.

- Low vision: a presenting visual acuity of better than $3 / 60$ but worse than $6 / 18$ in the better eye.

- Bilateral blindness: a presenting visual acuity of less than 3/60 in the better eye.

- Cataract blindness: a presenting visual acuity of less than 3/60 in the better eye, caused by lens opacity.

- Couching: any traditional form of treating cataract that does not involve non-touch technique.

- Cataract: refers to dense lens opacity that can explain the vision of less than 3/60.

- Trachoma: refers to dense trachomatous corneal opacity that can explain the visual loss of less than $3 / 60$.

- Glaucoma: defined as a cup/disc ratio of 0.9 or more, or if the media was not clear enough for optic disc assessment, a nonreactive pupil to light reflex in absence of definite causes like posterior synechiae.

- Post-intervention complications: refers to a blind eye with history of cataract surgery or couching, with the presumption that the poor vision was caused by the cataract surgery or couching procedure as evidenced by the presence of conditions like corneal decompensation, uveitis, etc.

- Uncorrected aphakia: refers to an aphakic eye whose vision improves to better than 3/60 with an aphakic glass ( $+10 \mathrm{D}$ glasses).

All records were processed and analysed in a computer database designed in EPI-INFO (version 6) software.

\section{Results}

In the 15 villages randomly selected, 1924 people were registered for the survey. A total of 1461 subjects were examined out of those that were registered. Thus, a participation rate of approximately $76 \%$ was achieved. The number of registered eligible people was less than the minimum sample size because some villages were smaller with fewer people aged 40 years and older.

AGE-SEX DISTRIBUTION OF SAMPLE POPULATION A total of 1461 people comprising 643 males (44\%) and 818 females (56\%) were examined. The age-sex distribution is presented in Table 1. 
Table 2 Distribution of subjects by visual status

\begin{tabular}{lcc}
\hline Vision & No of people & $\%(95 \%$ CI) \\
\hline Normal & 1239 & $84.8(81.2-88.5)$ \\
Low vision & 102 & $6.9(4.7-9.2)$ \\
Blind & 120 & $8.2(5.8-10.5)$ \\
Total & 1461 & 100.0 \\
\hline
\end{tabular}

Table 3 Causes of bilateral blindness

\begin{tabular}{lcr}
\hline Causes & No of people & \multicolumn{1}{l}{$\%$} \\
\hline Cataract & 53 & 44.2 \\
Corneal opacities & 19 & 15.8 \\
Post-intervention complications & 14 & 11.7 \\
Glaucoma & 7 & 5.8 \\
Uncorrected aphakia & 1 & 0.8 \\
Others & 26 & 21.7 \\
Total & 120 & 100.0 \\
\end{tabular}

PREVALENCE OF BLINDNESS

A total of 120 people were found to have a presenting vision of less than $3 / 60$ in the better eye. This corresponds to a blindness prevalence of $8.2 \%(95 \%$ CI $5.8 \%-10.5 \%)$ among the study population. The distribution of the sampled population by their visual status is contained in Table 2 .

CAUSES OF BLINDNESS

The major causes of bilateral blindness among the study population were cataract $(44.2 \%)$, corneal opacities $(15.8 \%)$, and postintervention complications (11.7\%). If blindness caused by cataract and that caused by intervention for cataract (couching or surgery) are added together it can be seen that cataract or cataract related causes are responsible for about $56 \%$ of all blindness. Table 3 shows the causes of bilateral blindness in the population.

CATARACT BLINDNESS

The prevalence of bilateral cataract blindness was found to be $3.6 \%$ (95\% CI $2.5 \%-5 \%)$ in the sampled population. There was no difference in the prevalence of cataract blindness between males and females; $\chi^{2}=0.11 \mathrm{p}=0.74$; relative risk (RR) of 1.14 (95\% CI 0.67-1.93). Table 4 shows the distribution of cataract blindness between the sexes.

CATARACT SURGICAL/COUCHING COVERAGE

A total of 38 people had aphakia in one or both eyes due to either cataract surgery or couching; 18 of these people had bilateral aphakia, while the remaining 20 individuals were found to have unilateral aphakia.

Seven people had cataract surgery while 31 people had couching in one or both eyes. As a whole 134 people were found to have cataract in one or both eyes. As such the couching coverage (people) is $18.0 \%$ (males $19.1 \%$, females $16.6 \%)$ and the cataract surgical coverage (people) is $4.0 \%$ (males $5.3 \%$, females $2.6 \%$ ) while the intervention (surgery + couching)

Table 4 Cataract blindness by sex

\begin{tabular}{llcl}
\hline Sex & $\begin{array}{l}\text { Cataract } \\
\text { blind }\end{array}$ & $\begin{array}{l}\text { Not cataract } \\
\text { blind }\end{array}$ & $\%(95 \% C I)$ \\
\hline Males & 25 & 618 & $3.8(2.3-6.3)$ \\
Females & 28 & 790 & $3.4(2.1-5.4)$ \\
Total & 53 & 1408 & $3.6(2.5-5.0)$ \\
\hline
\end{tabular}

Table 5 Types of complications in couched eyes

\begin{tabular}{lcc}
\hline Complications & No of eyes & $\%$ \\
\hline Corneal opacities & 14 & 37.8 \\
Dislocated lens (in view) & 11 & 28.9 \\
Uveitis/inflammation & 6 & 15.8 \\
Phthisis & 5 & 13.1 \\
Vitreous opacities & 2 & 5.3 \\
Total & 38 & 100 \\
\hline
\end{tabular}

Table 6 Barriers to cataract surgery

\begin{tabular}{lc}
\hline Barriers & $\begin{array}{l}\text { No of responses } \\
(\%)\end{array}$ \\
\hline Cannot afford treatment & $94(61.0)$ \\
Can see with the other eye & $29(18.8)$ \\
Not aware of place for treatment & $16(10.4)$ \\
God's wish & $7(4.5)$ \\
No trust & $3(1.9)$ \\
Distances & $2(1.3)$ \\
Prefers couching & $2(1.3)$ \\
No escort to treatment site & $1(0.6)$ \\
Total & $154(100)$ \\
\hline
\end{tabular}

coverage is $22.1 \%$ (males $24.4 \%$, females $19.2 \%$ ). Therefore about $78 \%$ of people with cataract in one or both eyes have not had either surgery or couching.

\section{CATARACT SURGICAL/COUCHING VISUAL} OUTCOMES

Surgical outcome

Nine eyes had cataract surgery in the hospital or free eye camps. Out of these three had poor visual outcome with resultant vision less than 3/60 even with aphakic spectacle correction. The complications identified as likely causes of the poor vision were chronic uveitis/ inflammation (in two eyes) and corneal decompensation (in one eye).

\section{Couching outcome}

Out of a total of 47 couched eyes only nine had a vision of $3 / 60$ or better with $+10 \mathrm{D}$ glasses.

This indicates that only $26.8 \%$ of eyes that had couching could see $3 / 60$ or better. Table 5 shows the form of complications identified among couched eyes.

\section{BARRIERS TO CATARACT SURGERY UPTAKE}

Many people with cataract or "couched eye" reported more than one factor responsible for not having surgery. One common reason, however, mentioned by most is the inability to afford the treatment $(61 \%)$. In addition some respondents did not know where they could get treatment even if they could afford it (10\%). Virtually all the people with unilateral cataract also reported that they see at least with the other eye. Table 6 shows the identified barriers to uptake of cataract services.

\section{Discussion}

Only $61 \%$ of the minimum sample size of 2396 or $76 \%$ of registered population were examined. This could be attributed to the fact that about half of the villages visited had a smaller population than depicted in the census data; consequently the villages had inadequate numbers of the eligible population for examination (that is, 170 people 40 years and above). The survey was also conducted during the nonplanting season, a period when the healthy 
male adults in the villages (ages 20-50) travel to the cities for temporary jobs. This may explain why only $76 \%$ of registered eligible people in the villages were examined.

The high prevalence of blindness among 40 years and over $(8.2 \%)$ in this district may not be surprising for a remote, underserved rural area of a developing country. In this subdistrict of Nigeria cataract services, and indeed all eye care services, are not available. Where such services are available, they are not only inaccessible but also unaffordable to the majority of the population. In the Indian rural community of Mohadi block of Bhandara district of Maharashtra State, a blindness prevalence of $6.8 \%$ was found among 40 years and above. ${ }^{6}$ In Nepal a survey of those aged 45 years and above reported a blindness prevalence of $3.77 \%{ }^{7}$

The second major cause of blindness after cataract in the study area was corneal opacities (responsible for $15.8 \%$ of blindness). This is believed to be mainly due to trachoma, as the area lies in the WHO (World Health Organization) trachoma endemic belt of northern Nigeria. ${ }^{8}$ A recently conducted survey in the area has reported a prevalence of trichiasis among adult females as $8.6 \%$ and prevalence of active trachoma among children of $11.1 \% .^{9}$ This means that trachoma is a public health problem in this area. Blindness from postintervention complications $(11.7 \%)$ may have been overestimated since the cause of visual loss, which is attributed to the intervention in this study, may actually have predated the intervention. The causes of blindness attributed to glaucoma $(5.8 \%)$ in the study may have been underestimated because the case definition of blindness was only based on visual acuity with no visual field blindness criteria.

The high prevalence of cataract blindness $(3.6 \%)$ in this subdistrict is not unexpected in view of the lack of regular cataract services in this remote rural area. It is worth mentioning that even if regular services were made available the issue of affordability among this poor rural population would still be a potentially limiting factor in uptake of the services. Studies in rural settings of other regions of the world have reported similar values of cataract blindness. In the Karnataka state of India the age and sex adjusted prevalence of cataract blindness among those 50 years and older was found to be $4.93 \%$ with a range of $1.58 \%$ to $7.24 \%$ in different districts. ${ }^{10}$ In the Mohadi block of Bhandara, Maharashtra state of India a prevalence of $6.2 \%$ of cataract blindness amongst 40 years and older was found. ${ }^{6}$

A cataract surgical coverage for people of $4.1 \%$ and a couching coverage for people of $18.0 \%$ meant that couching was being practised about four times more frequently than cataract surgery in this area. However, $78 \%$ of the people with cataract blindness in at least one eye have not had any treatment for it (in terms of either cataract surgery or couching). But it could be argued that these values may be an exaggeration since the couching recorded in this study was presumed to have been done on cataract blind eyes only, which may not be so. The couching might have been done on an eye that neither had cataract nor was blind at the time of the couching. Also the cataract surgery might have been done not necessarily on a blind eye. The low level of cataract surgical coverage is likely to be due to the nonavailability of cataract services in the area or, at best, the cataract services are very irregular. Even when the services are available most people cannot afford the charges, as they are very poor, living essentially by subsistence farming. Moreover, some of the villages are inaccessible for a part of the year (as in the rainy season). Furthermore, the outcome of some cataract surgeries has not inspired confidence in the operation. This is an important consideration in an area where couching is also performed. As such people make do with what is readily available and affordable to them-that is, couching. Even though the outcome of couching is mostly unfavourable many people in this subdistrict seem to have no choice other than to take a chance with couching.

As in most surveys on the barriers to uptake of cataract services, the financial limitations of the people ranked first, constituting over $60 \%$ of responses as the reason for not having cataract surgery. Although most respondents gave more than one reason, the issue of cost was the most frequently mentioned. The summation of the direct and indirect costs of obtaining cataract services is excessively beyond the ability of the poor peasant subsistent farmers, who participated in this study. About 16 people $(10 \%)$ reported that they were not aware of a place to seek treatment even if they had the money to pay for the treatment. This may be as a result of the non-availability of cataract services nearby coupled with a non-functional primary healthcare system which, in an improved state of affairs, could provide the necessary referral for treatment, in addition to other health promotion and education activities. It is noteworthy that three people $(2 \%)$ expressed their distrust of surgery. These people have all had surgery in one eye with poor visual outcome.

Cost, lack of information, "can see with other eye," and fear of operation are the main factors identified in most studies as the barriers to uptake of cataract services. Studies by Johnson et al in Gambia, ${ }^{11}$ Limburg and Kumar in Karnataka state of India, ${ }^{10}$ Snelligen et al in Nepal, ${ }^{12}$ and Limburg et al in Maharashtra state of India ${ }^{6}$ all reported similar barriers.

Some of the limitations/constraints of this study included the choice of a cluster size of 170 , which is too big for a cluster random sampling survey. The limited time and resources for the survey necessitated this. The choice of a smaller cluster size (which would have been desirable) would have meant visiting more villages and thus consume more time and resources for the survey. Another major limitation encountered was the unreliable census data used for the design of the survey. This had affected the study design and the choice of the cluster size, which resulted partially in the less than optimal coverage encountered in the survey. Furthermore, the dearth of complete age and sex population data of the study area did 
not allow calculation of age and sex adjusted prevalence rates.

\section{Conclusion}

The findings suggest that the study area has a high burden of cataract blindness. This may be attributed mainly to the non-availability of regular cataract services in the region, coupled with the inability of the peasant rural people to afford treatment elsewhere. It is thus necessary to set up measures to address this problem. Cataract surgical services should be readily available and affordable to the rural people, who form the vast majority of the populace in this part of the world. A free cataract outreach programme for such areas will be ideal.

This work was supported by the "Foundation Dark and Light" of the Netherlands. I wish to thank Professor Adenike Abiose of the National Eye Centre Kaduna, Nigeria, Dr Allen Foster of the London School of Hygiene and Tropical Medicine, and Brenden Dineen of the International Centre for Eye Health
London for their contributions in the design conduct and London for their contributions in the design conduct and
reporting of this work. I am also grateful to the Katsina state government and the whole field team for their support.

1 World Health Organization. Blindness and visual disability. Part IV of VII. WHO fact sheet. Geneva: WHO, $1997 \mathrm{Feb}$ No145:2.
2 Fletcher A. Barriers to using eye services and recommendation to improve service uptake. Research finding and international workshop recommendation. Madurai, India 1998:10.

3 Limburg H, Kumar R, Indrayan A, et al. Rapid assessment of prevalence of cataract blindness at district level. Int $\mathcal{F}$ Epidemiol 1997;26:1049-54.

4 National Population Commission reports. Abuja, Nigeria, 1997.

5 Lawan A. Prevalence and causes of blindness and visual impairment in Dambatta Local Government Area of Kano State, Nigeria. Fellowship dissertation. National Post-graduate Medical College of Nigeria, Lagos, 1995: 45.

6 Limburg H, Vaidyanathan K, Pampattiwar KN. Cataract blindness on the rise? Results of a door-to-door examination in Mohadi. Indian f Ophthalmol 1996; 44:241-2.

7 Pokharel GP, Regin G, Shrestha SK, et al. Prevalence of blindness and cataract surgery in Nepal. Br f Ophthalmol 1998;82:600-5.

8 World Health Organization. Future approaches to trachoma control. Report of a global scientific meeting. WHO/PBL/ 96.50:4. Geneva: WHO, 1996.

9 Rabiu MM. Prevalence of trachoma and its risk factors in a rural community of northern Nigeria. Fellowship dissertation. National Postgraduate Medical College of Nigeria, Lagos, 2000:27.

10 Limburg H, Kumar R. Follow up study of blindness attributed to cataract in Karnataka State India. Ophthalmic Epidemiol 1998;5 211-23.

11 Johnson JG, Goode V, Faal H. Barriers to the uptake of cataract surgery. Tropical Doctor 1998;28:218-20.

12 Snelligen T, Shrestha BR, Gharti MP, et al. Socioeconomic barriers to cataract surgery in Nepal: the South Asian cataract management study. $\mathrm{Br} f \mathrm{f}$ Ophthalmol 1998;82: $1424-8$ 